\title{
Delayed allergic reaction to amlodipine with a positive lymphocyte transformation test
}

\section{Reacción alérgica tardía a amlodipino con positividad de la prueba de transformación de linfocitos}

\author{
Olga Patricia Monge-Ortega, ${ }^{1}$ Javier Domínguez-Ortega, ${ }^{2,3}$ Miguel González-Muñoz, ${ }^{5}$ Rosario Cabañas, ${ }^{2,4}$ \\ Magdalena Lluch-Bernal, ${ }^{2}$ Ana Fiandor, ${ }^{2,4}$ Luz Yadira Bravo-Gallego, ${ }^{5}$ Santiago Quirce ${ }^{2,3}$
}

\begin{abstract}
Background: Amlodipine is one of the third generation dihydropyridine calcium channel blockers used for hypertension. Mild, moderate and severe reactions have been reported with calcium channel blockers. Cross-reactivity among these drugs has not been established.

Case report: We have presented the case of a patient who developed a delayed pruritic, and erythematous maculopapular skin exanthema after the intake of amlodipine. A positive lymphocyte transformation test (LTT) confirmed the implication of amlodipine in the reaction and showed positivity to another calcium channel blocker, nifedipine, demonstrating cross reactivity.

Conclusion: This is the first reported case of hypersensitivity to amlodipine in which the involvement of the drug is confirmed by a positive TTL. TTL could become a good diagnostic alternative for patients who experience late reactions to amlodipine and possibly cross-reactivity with nifedipine.
\end{abstract}

Keywords: Allergy; Amlodipine; Delayed reaction; Lymphocyte transformation test

Este artículo debe citarse como: Monge-Ortega OP, Domínguez-Ortega J, González-Muñoz M, Cabañas R, Lluch-Bernal M, Fiandor A, Bravo-Gallego LY, Quirce S. Reacción alérgica tardía a amlodipino con positividad de la prueba de transformación de linfocitos. Rev Alerg Mex 2017;64(4):505-508

${ }^{1}$ Hospital San Juan de Dios, Departamento de Alergia. San José, Costa Rica

${ }^{2}$ Hospital La Paz, Departamento de Alergia. Madrid, España

${ }^{3}$ Centro de Investigación Biomédica en Red de Enfermedades

Respiratorias. Madrid, España

${ }^{4}$ Consorcio PielenRED. Madrid, España

${ }^{5}$ Hospital La Paz, Departamento de Inmunología. Madrid, España

\begin{abstract}
Correspondencia: Olga Patricia Monge-Ortega. patmonor@hotmail.com
\end{abstract}

Recibido: 2017-09-01

Aceptado: 2017-11-03 


\section{Resumen}

Antecedentes: el amlodipino es uno de los bloqueadores de los canales de calcio de dihidropiridinas de tercera generación utilizado para la hipertensión. Se han notificado reacciones leves, moderadas y graves con bloqueadores de los canales de calcio. No se ha establecido la reactividad cruzada entre estos fármacos.

Caso clínico: se presenta el caso de una paciente con exantema cutáneo maculopapular, eritematoso y pruriginoso tardío tras la ingestión de amlodipino. La prueba de transformación de linfocitos mostró positividad al amlodipino, lo que confirmó la implicación de este fármaco en la reacción; también mostró positividad al nifedipino, lo que demostró la presencia de reactividad cruzada.

Conclusión: este es el primer caso reportado de hipersensibilidad a amlodipino en el que se confirma la implicación del fármaco por medio de una prueba positiva de transformación de linfocitos, la cual podría convertirse en una buena opción diagnóstica en aquellos pacientes que experimentan reacciones tardías al amlodipino, así como posible reactividad cruzada con nifedipino.

Palabras clave: Alergia; Amlodipino; Reacción tardía; Prueba de transformación linfocitaria

\author{
Abreviaturas y siglas \\ $\mathrm{CCB}$, bloqueadores de canales de calcio de dihidropi- \\ ridina
}

LTP, proteínas de transporte de lípidos

SI, índice de estimulación

TTL, prueba de transformación de linfocitos

\section{Antecedentes}

El amlodipino es un fármaco de los bloqueadores de los canales de calcio de dihidropiridinas de tercera generación (CCB) utilizado para el control de la hipertensión; inhibe la afluencia de iones de calcio a través de los canales de calcio tipo L de los músculos lisos vasculares, causando vasodilatación. Por lo general se prescribe una vez al día debido a su vida media prolongada. Tiene alta biodisponibilidad que alcanza 60 a $80 \%$, con un tiempo de eliminación entre 40 y 60 horas.

El edema periférico es el efecto adverso que se reporta con más frecuencia; otros son enrojecimiento, mareo, astenia, cefalea, palpitaciones, vértigo y náuseas. ${ }^{1}$ Se ha descrito angioedema en pacientes que reciben antagonistas de los canales de calcio, ${ }^{2}$ así como casos aislados de necrosis epidérmica tóxica, ${ }^{3}$ penfigoide ampolloso con eritema multiforme, ${ }^{4}$ mucositis oral y síndrome de Steven-Johnson. ${ }^{5}$ Las reacciones de hipersensibilidad asociadas con amlodipino ocurren con menos frecuencia que con otros bloqueadores de los canales de calcio, como el diltiazem. No se ha establecido bien la reactividad cruzada entre estos medicamentos. ${ }^{6}$

\section{Caso clínico}

Mujer de 55 años de edad, de origen español, atendida en el Hospital Universitario La Paz, Madrid, España, con antecedente de hipertensión y dislipidemia. Su médico de atención primaria le prescribió amlodipino, que constituyó el primer fármaco antihipertensivo que utilizaba. Un mes después desarrolló lesiones cutáneas maculopapulares y pruriginosas en la región distal y anterolateral de ambos miembros inferiores, asociadas con edema local, motivo por el cual fue evaluada por un dermatólogo en el Departamento de Emergencias; los exámenes de laboratorio séricos resultaron normales, incluida la ausencia de eosinofilia en sangre. Por sospecha de que las manifestaciones clínicas tuvieran relación con el fármaco, se le recomendó que lo interrumpiera y se prescribieron antihistamínicos 
orales y corticosteroides tópicos. La paciente ya no mostró síntomas después de 15 días de tratamiento. El amlodipino se sustituyó por enalapril, sin nuevas incidencias.

Tres meses después, en el Departamento de Alergia del mismo hospital, las pruebas intraepidérmicas (prick test) resultaron negativas para extractos comerciales de los aeroalérgenos comunes en la zona, principales alimentos, Anisakis simplex, látex, a un extracto de proteínas de transporte de lípidos estandarizadas (LTP) de melocotón (ALK ${ }^{\circledR}$, Madrid, España y LETI Unipersonal ${ }^{\circledR}$, Barcelona. España) y para amlodipino $(5 \mathrm{mg} / \mathrm{mL})$. También fueron negativas las pruebas de parche con una serie true-test estándar (SmartPractice Denmark ApS) y amlodipino, utilizando un comprimido pulverizado para obtener una concentración de $30 \%$ en vaselina. ${ }^{7}$

Para aclarar el mecanismo subyacente, 5 meses después de la reacción se realizó una prueba de transformación de linfocitos (TTL) con amlodipino y nifedipino (otro fármaco bloqueador de los canales de calcio al que la paciente tenía acceso y que podía ser otra opción para tratar la hipertensión arterial en el futuro). Se realizó la medición de la proliferación de linfocitos, tomando en cuenta la utilidad de este parámetro. ${ }^{8}$ Se incubaron células mononucleares de sangre periférica frescas y separadas sobre un gradiente de densidad (Histopaque ${ }^{\circledR}-1077$, Sigma-Aldrich, EUA) durante 6 días a $10^{6}$ células $/ \mathrm{mL}$, en triplicado, con amlodipino (50 $\mu \mathrm{g} / \mathrm{mL}-1 \mu \mathrm{g} / \mathrm{mL})$ y nifedipino $(200 \mu \mathrm{g} / \mathrm{mL}-1 \mu \mathrm{g} /$ $\mathrm{mL})$. Se utilizó fitohemaglutinina $(5 \mu \mathrm{g} / \mathrm{mL})$ como control positivo. La proliferación se determinó mediante la adición de $[3 \mathrm{H}]$ timidina $(0.5 \mu \mathrm{Ci} /$ pocillo $)$ durante las últimas 18 horas del periodo de incubación. La respuesta proliferativa se calculó con el índice de estimulación (SI), definido como la relación entre los valores medios de los recuentos por minuto del antígeno en cultivos y los obtenidos sin el antígeno. De amlodipino y nifedipino se obtuvo una respuesta positiva, definida como un $\mathrm{SI}>2$. En tres sujetos control, las TTL con amlodipino y nifedipino no mostraron respuestas proliferativas (Cuadro 1).

Como la paciente se negó a someterse a otra prueba in vivo, no se realizó prueba de exposición oral con amlodipino o nifedipino. La paciente continuó con buena tolerancia a omeprazol y atorvastatina como parte de su tratamiento habitual para sus comorbilidades crónicas.

\section{Discusión}

En la paciente descrita, la TTL mostró positividad que confirmó la implicación del amlodipino en la reacción alérgica; también mostró positividad a otro bloqueador de los canales de calcio, el nifedipino, lo cual demostró reactividad cruzada.

La TTL ha sido utilizada para evaluar la reacción alérgica tardía. ${ }^{9,10}$ Las ventajas que ofrece respecto al parche y las pruebas intradérmicas incluyen la completa seguridad del paciente y la evaluación de la respuesta de células $\mathrm{T}$ al fármaco, especialmente cuando se realiza de 3 a 9 meses después de que se inició la reacción. Al revisar la literatura internacional no identifica-

\begin{tabular}{|c|c|c|c|c|c|c|}
\hline \multicolumn{7}{|c|}{ Índice de estimulación } \\
\hline \multicolumn{4}{|c|}{ Amlodipino $(\mu \mathrm{g} / \mathrm{mL})$} & \multicolumn{3}{|c|}{ Nifedipino $(\mu \mathrm{g} / \mathrm{mL})$} \\
\hline & 50 & 10 & 1 & 200 & 100 & 10 \\
\hline Paciente & 0.8 & 2.2 & 1.8 & 4.8 & 1.2 & 1.1 \\
\hline Control 1 & 1.2 & 1.1 & 0.8 & 0.7 & 1.2 & 0.8 \\
\hline Control 2 & 0.5 & 1.1 & 1.0 & 0.8 & 1.5 & 1.5 \\
\hline Control 3 & 0.7 & 0.9 & 0.8 & 1.6 & 1.9 & 1.0 \\
\hline
\end{tabular}

Resultados de TTL a diferentes concentraciones de amlodipino y nifedipino. Se expresan como un índice de estimulación media de cultivos triplicados 
mos otro caso reportado de hipersensibilidad a amlodipino en el que se confirme la implicación del fármaco mediante una TTL, la cual podría convertirse en una buena alternativa diagnóstica en aquellos pacientes que experimentan reacciones tardías al amlodipino con posible reactividad cruzada con nifedipino.
Financiamiento

Los autores declaran que no se recibieron fondos para el presente estudio.

\section{Conflicto de interés}

Los autores declaran que no tienen conflictos de interés para la publicación de este caso clínico.

\section{Referencias}

1. Fares H, DiNicolantonio JJ, O'Keefe JH, Lavie CJ. Amlodipine in hypertension: A first-line agent with efficacy for improving blood pressure and patient outcomes. Open Heart. 2016;3(2):e000473. DOI: http://dx.doi.org/10.1136/openhrt-2016-000473

2. Southward J, Irvine E, Rabinovich M. Probable amlodipine-induced angioedema. Ann Pharmacother. 2009;43(4):772-776. DOI: http://dx.doi.org/10.1345/aph.1L527

3. Baetz BE, Patton ML, Guiday RE, Reigart CL, Ackerman BH. Amlodipine-induced toxic epidermal necrolysis. JBurn Care Res.2011;32(5):158-160.DOI:http://dx.doi.org/10.1097/BCR.0b013e31822ac7be

4. Park KY, Kim BJ, Kim MN. Amlodipine-associated bullous pemphigoid with erythema multiforme-like clinical features. Int J Dermatol. 2011;50:637-639. DOI: http://dx.doi.org/10.1111/j.1365-4632.2009.04417.x

5. Wiwanitkit S, Wiwanitkit V. Oral mucositis, Steven Johnson syndrome and amlodipine usage in a case with metabolic syndrome. Diabetes Metab Syndr. 2012;6(2):112. DOI: http://dx.doi.org/10.1016/j. dsx.2012.08.016

6. Gonzalo-Garijo MA, Pérez-Calderón R, De-Argila-Fernández-Durán D, Rangel-Mayoral JF. Cutaneous reactions due to diltiazem and cross reactivity with other calcium channel blockers. Allergol Immunopathol (Madr). 2005;33(4):238-240. Disponible en: https://pdfs.semanticscholar.org/c51a/1213bf42340b2603d 5d93f16a977793f889a.pdf

7. Pichler WJ, editor. Drug hypersensitivity. Suiza: Karger; 2007.

8. Ariza A, Montañez MI, Fernández TD Perkins JR, Mayorga C. Cellular tests for the evaluation of drug hypersensitivity. Curr Pharm Des. 2016;22(45): 6773-6783. DOI: http://dx.doi.org/10.2174/1381612822 666161107165917

9. Domínguez-Ortega J, Entrala A, Pola-Bibian B, González-Muñoz M, Fiandor A, Quirce S. Delayed allergic reaction to acenocoumarol with a positive lymphocyte transformation test. J Investig Allergol Clin Immunol. 2016;26(4):273-275. DOI: http://dx.doi.org/10.18176/jiaci.0072

10. González-Cavero L, Domínguez-Ortega J, González-Muñoz M, Mayor-lbarguren A, Tomás M, Fiandor $A$, et al. Delayed allergic reaction to terbinafine with a positive lymphocyte transformation test. J Invest Allergol Clin Immunol. 2017;27(2):136-137. DOI: http://dx.doi.org/10.18176/jiaci.0133 